\title{
The CALorimetric Electron Telescope (CALET) on the International Space Station: Results from the First Two Years of Operation
}

\author{
$Y$ Asaoka $^{1,2, *}, O$ Adriani $^{3,4}, Y$ Akaike $^{5,6}, K$ Asano $^{7}, M G$ Bagliesi $^{8,9}, E$ Berti $^{3,4}, G$ Bigongiari $^{8,9}, W R$ Binns $^{10}, S$ Bonechi $^{8,9}$, \\ $M$ Bongi $^{3,4}, A$ Bruno ${ }^{11}, P$ Brogi $^{8,9}, J H$ Buckley $^{10}, N$ Cannady $^{12}, G$ Castellini ${ }^{13}, C$ Checchia $^{14,15}, M L$ Cherry $^{12}$, \\ $G$ Collazuol $^{14,15}, V D i$ Felice $^{16,17}, K$ Ebisawa $^{18}, H$ Fuke $^{18}, T G$ Guzik $^{12}, T$ Hams $^{5,19}, N$ Hasebe ${ }^{1}, K$ Hibinov $^{20}$, \\ $M$ Ichimura $^{21}, K$ Ioka $^{22}, W$ Ishizaki $^{7}, M H$ Israel $^{10}, K$ Kasahara $^{1}, J \mathrm{Kataoka}^{1}, R$ Kataoka ${ }^{23}, Y \mathrm{Katayose}^{24}, C \mathrm{Kato}^{25}$, \\ $N$ Kawanaka $^{26,27}, Y$ Kawakubo $^{28}, K$ Kohri $^{29}, H S$ Krawczynski $^{10}, J F$ Krizmanic $^{19,5}, T$ Lomtadze $^{9}, P$ Maestro $^{8,9}$, \\ $P S$ Marrocchesi $^{8,9}, A M$ Messineo $^{30,9}, J W$ Mitchell $^{6}, S$ Miyake $^{31}, A A$ Moiseev $^{32,19}, K$ Mori $^{1,18}, M$ Mori $^{33}, N$ Mori $^{4}$, \\ $H M$ Motz $^{34}, K$ Munakata $^{25}, H$ Murakami $^{1}, S$ Nakahira $^{35}, J$ Nishimura $^{18}, G A$ De Nolfo ${ }^{11}, S$ Okuno $^{20}, J F$ Ormes $^{36}$, \\ $S$ Ozawa $^{1}, L$ Pacini $^{3,13,4}, F$ Palma ${ }^{16,17}, V$ Pal'shin ${ }^{28}, P$ Papini $^{4}, A V$ Penacchioni $^{8,37}, B F$ Rauch $^{10}, S B$ Ricciarini $^{13,4}$, \\ $K$ Sakai $^{19,5}, T$ Sakamoto $^{28}, M$ Sasaki $^{19,32}, Y$ Shimizu $^{20}, A$ Shiomi ${ }^{38}, R$ Sparvoli $^{16,17}, P$ Spillantini ${ }^{3}, F$ Stolzi $^{8,9}, S$ Sugita $^{28}$,

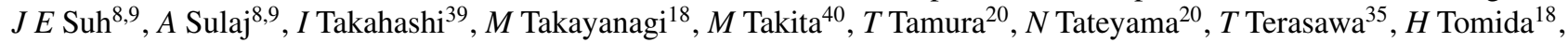 \\ $S$ Torii $^{1,41}, Y$ Tsunesada $^{42}, Y$ Uchihori $^{43}, S$ Ueno $^{18}, E$ Vannuccini $^{4}, J P$ Wefel $^{12}, K$ Yamaoka $^{44}, S$ Yanagita $^{45}, A$ Yoshida $^{28}$, \\ and $K$ Yoshida $^{46}$
}

${ }^{1}$ Waseda Research Institute for Science and Engineering, Waseda University, Japan

${ }^{2}$ JEM Utilization Center, Human Spaceflight Technology Directorate, Japan Aerospace Exploration Agency, Japan

${ }^{3}$ Department of Physics, University of Florence, Italy

${ }^{4}$ INFN Sezione di Florence, Italy

${ }^{5}$ Department of Physics, University of Maryland, USA

${ }^{6}$ Astroparticle Physics Laboratory, NASA/GSFC, USA

${ }^{7}$ Institute for Cosmic Ray Research, The University of Tokyo, Japan

${ }^{8}$ Department of Physical Sciences, Earth and Environment, University of Siena, Italy

${ }^{9}$ INFN Sezione di Pisa, Italy

${ }^{10}$ Department of Physics, Washington University, USA

${ }^{11}$ Heliospheric Physics Laboratory, NASA/GSFC, USA

${ }^{12}$ Department of Physics and Astronomy, Louisiana State University, USA

${ }^{13}$ Institute of Applied Physics (IFAC), National Research Council (CNR), Italy

${ }^{14}$ Department of Physics and Astronomy, University of Padova, Italy

${ }^{15}$ INFN Sezione di Padova, Italy

${ }^{16}$ University of Rome "Tor Vergata”, Italy

${ }^{17}$ INFN Sezione di Rome "Tor Vergata", Italy

${ }^{18}$ Institute of Space and Astronautical Science, Japan Aerospace Exploration Agency, Japan

${ }^{19}$ CRESST and Astroparticle Physics Laboratory NASA/GSFC, USA

${ }^{20}$ Kanagawa University, Japan

${ }^{21}$ Faculty of Science and Technology, Graduate School of Science and Technology, Hirosaki University, Japan

${ }^{22}$ Yukawa Institute for Theoretical Physics, Kyoto University, Japan

${ }^{23}$ National Institute of Polar Research, Japan

${ }^{24}$ Faculty of Engineering, Division of Intelligent Systems Engineering, Yokohama National University, Japan

${ }^{25}$ Faculty of Science, Shinshu University, Japan

${ }^{26}$ Hakubi Center, Kyoto University, Japan

${ }^{27}$ Department of Astronomy, Graduate School of Science, Kyoto University, Japan

${ }^{28}$ College of Science and Engineering, Department of Physics and Mathematics, Aoyama Gakuin University, Japan

${ }^{29}$ Institute of Particle and Nuclear Studies, High Energy Accelerator Research Organization, Japan

${ }^{30}$ University of Pisa, Italy

${ }^{31}$ Department of Electrical and Electronic Systems Engineering, National Institute of Technology, Ibaraki College, Japan

${ }^{32}$ Department of Astronomy, University of Maryland, USA

${ }^{33}$ Department of Physical Sciences, College of Science and Engineering, Ritsumeikan University, Japan

${ }^{34}$ Global Center for Science and Engineering, Faculty of Science and Engineering, Waseda University, Japan

${ }^{35}$ RIKEN, Japan

${ }^{36}$ Department of Physics and Astronomy, University of Denver, USA

${ }^{37}$ ASI Science Data Center (ASDC), Italy

${ }^{38}$ College of Industrial Technology, Nihon University, Japan

${ }^{39}$ Kavli Institute for the Physics and Mathematics of the Universe, The University of Tokyo, Japan 
${ }^{40}$ Institute for Cosmic Ray Research, Japan

${ }^{41}$ School of Advanced Science and Engineering, Waseda University, Japan

${ }^{42}$ Division of Mathematics and Physics, Graduate School of Science, Osaka City University, Japan

${ }^{43}$ National Institutes for Quantum and Radiation Science and Technology, JAPAN

${ }^{44}$ Nagoya University, Japan

${ }^{45}$ College of Science, Ibaraki University, Japan

${ }^{46}$ Department of Electronic Information Systems, Shibaura Institute of Technology, Japan

\begin{abstract}
The CALorimetric Electron Telescope (CALET) space experiment, which has been developed by Japan in collaboration with Italy and the United States, is a high-energy astroparticle physics mission on the International Space Station (ISS). The primary goals of the CALET mission include investigation of possible nearby sources of high-energy electrons, detailed study of galactic cosmic-ray acceleration and propagation, and search for dark matter signatures. With a long-term observation onboard the ISS, the CALET experiment measures the flux of cosmic-ray electrons (including positrons) up to $20 \mathrm{TeV}$, gamma-rays to $10 \mathrm{TeV}$, and nuclei up to $1,000 \mathrm{TeV}$ based on its charge separation capability from $Z=1$ to 40 . Since the start of science operation in mid-October, 2015, a continuous observation has been maintained without any major interruptions. The number of triggered events over $10 \mathrm{GeV}$ is nearly 20 million per month. By using the data obtained during the first two-years, here we present a summary of the CALET observations: 1) Electron+positron energy spectrum, 2) Nuclei analysis, 3) Gamma-ray observation with a characterization of the on-orbit performance. The search results for the electromagnetic counterparts of LIGO/Virgo gravitational wave events are also discussed.
\end{abstract}

\section{Introduction}

The CALorimetric Electron Telescope (CALET) [1, 2], which has been developed by Japan in collaboration with Italy and the United States, is a high-energy astroparticle physics experiment installed on the International Space Station (ISS). The CALET detector was launched on August 19,2015 by a Japanese H2-B rocket as part of the payload of an unmanned H2 Transfer Vehicle (HTV) resupplying the ISS. It was then installed on the Japanese Experiment Module-Exposed Facility (JEM-EF) of the ISS, as shown in Fig. 1, for a two-year mission, extendable to five years (or more). CALET is attached at the port \#9 on the JEM-EF, where a mostly unobstructed field-of-view of $45^{\circ}$ from the zenith is available. A schematic overview of the CALET payload is presented in the lower part of Fig. 1. The total weight is $613 \mathrm{~kg}$ and the maximum power consumption is $507 \mathrm{~W}$.

CALET features a very thick calorimeter that incorporates imaging and total absorption calorimeters. The overall thickness of CALET at normal incidence is 30 radiation length $\left(X_{0}\right)$ and $\sim 1.3$ proton interaction length. Long-term observation using a large-area detector is possible by observation onboard the ISS. CALET's primary objective is to discover nearby cosmic-ray accelerators and search for dark matter by precisely measuring the all-electron (electron + positron) and gamma-ray spectra in a wide energy range from $1 \mathrm{GeV}$ to $20 \mathrm{TeV}$. The main components of cosmic rays, such as protons, helium, and nuclei through iron, can be measured in the range up to a PeV. The details of propagation and acceleration of the galactic cosmic-rays will be studied based on the nuclei spectra. It is expected that CALET will perform unique observations by extending the previous limits of direct measurements.

\section{Detector}

The CALET calorimeter, shown in side view in Fig. 2 along with a simulation of a $1 \mathrm{TeV}$ electron shower, has

\footnotetext{
*e-mail: yoichi.asaoka@aoni.waseda.jp
}

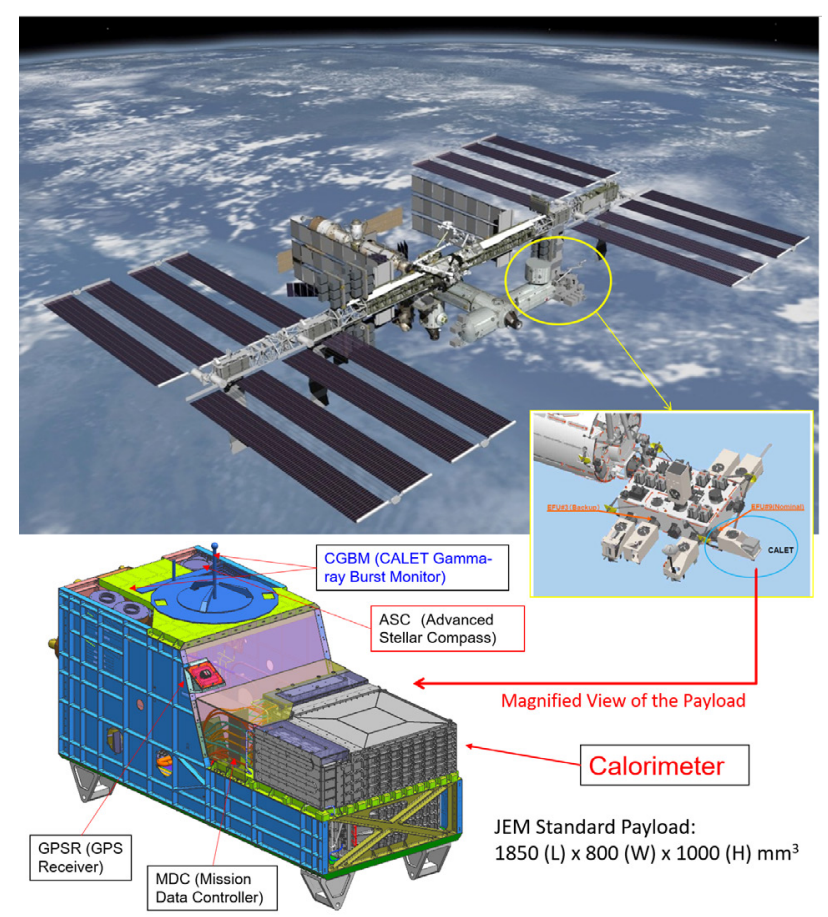

Figure 1. The CALET payload $[1,2]$ onboard the ISS as part of the Japanese Experiment Module - Exposed Facility. The payload includes the main calorimeter, CALET Gamma-ray Burst Monitor (CGBM) subsystems [3], Mission Data Controller (MDC), and two support sensors consisting of Advanced Stellar Compass (ASC) and Global Position Sensor Receiver (GPSR) as indicated in the figure.

several unique and important characteristics, as briefly mentioned in the Introduction. These include its ability to resolve in detail the initial development of showers, as well as tracks generated by non-interacting minimum ionizing particles (MIPs), and its capacity to precisely measure the energy of electrons in the $\mathrm{TeV}$ region as a result of its depth of $30 X_{0}$. These features are achieved through a combina- 


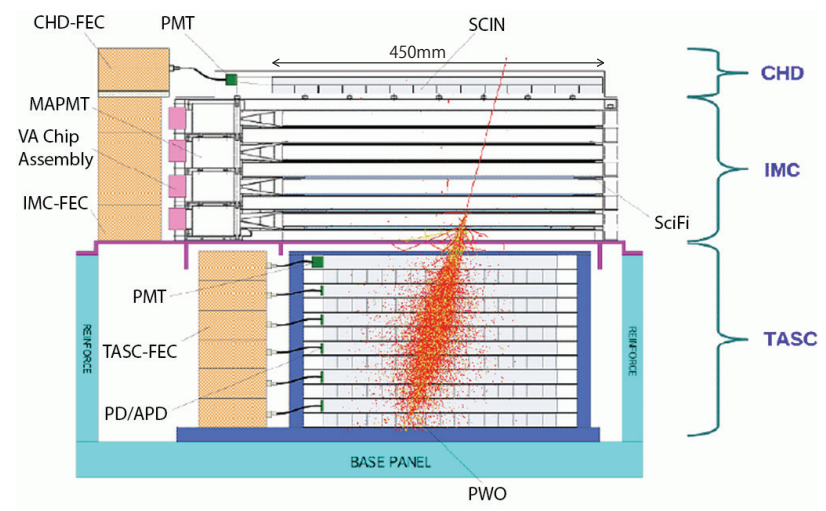

Figure 2. CALET calorimeter $[1,2]$ consisting of three sub detectors, i.e., a CHarge Detector (CHD), an IMaging Calorimeter (IMC), and Total AbSorption Calorimeter (TASC).

tion of three primary detector sub-systems: Particle identification and energy measurements are performed by the TASC, charge identification is obtained from a charge detector (CHD), and an imaging calorimeter (IMC) is employed for track reconstruction. The key performance of each detector component, as described below, was estimated on the basis of a detailed Monte Carlo (MC) simulation and was confirmed by several beam tests carried out primarily at the CERN-SPS facility.

Plastic scintillators arranged in two orthogonal layers, each containing 14 scintillator paddles $(3.2 \times 1.0 \times 45.0$ $\mathrm{cm}^{3}$ ), constitute the CHD. These paddles generate photons that are detected by a photomultiplier tube (PMT), and the resulting output is sent to a front end circuit (FEC). This FEC and the subsequent readout system have sufficient dynamic range for particle charges in the range of $Z=1 \sim 40$.

The initial shower is resolved by the IMC sampling calorimeter, which has been carefully designed to accurately determine the shower starting point and incident direction. This calorimeter has a thickness of $3 X_{0}$ and contains five upper $0.2 X_{0}$ and two lower $1.0 X_{0}$ tungsten plates. The IMC contains a total of 16 detection layers, arranged in $8 \mathrm{X}$-Y pairs, with each layer segmented into 448 parallel scintillating fibers $\left(0.1 \times 0.1 \times 44.8 \mathrm{~cm}^{3}\right)$, which are individually read out by 64-channel multi anode PMTs.

The TASC has an overall depth of $27 X_{0}$ and consists of 12 detection layers in an alternating orthogonal arrangement, each comprised of 16 lead tungstate crystal $\left(\mathrm{PbWO}_{4}\right.$ or PWO) $\operatorname{logs}$ with dimensions of $2.0 \times 1.9 \times 32.6 \mathrm{~cm}^{3}$. As a result of this design, the TASC is able to image the development of the shower in three dimensions. With the exception of the first layer which uses PMTs, a photodiode (PD) in conjunction with an avalanche photodiode (APD) reads the photons generated by each PWO log. Employing dual shaping amplifiers with two different gains for each APD (PMT) and PD, increases the dynamical range to $10^{6}$ $\left(10^{4}\right)$.

Combining these components as well as the trigger system, data acquisition system and support sensors, the CALET instrument features (1) a proton rejection factor of

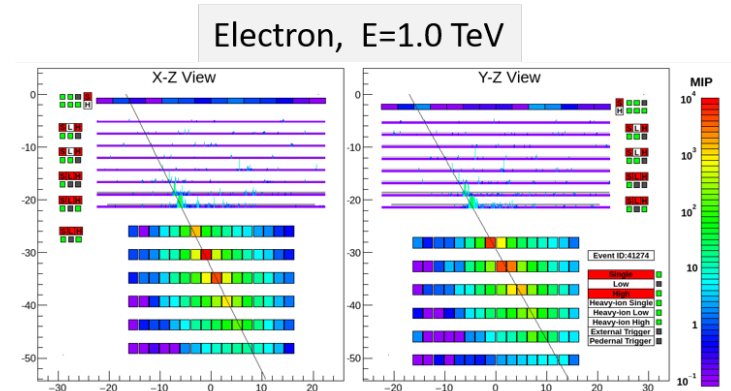

Proton, $\Delta \mathrm{E}=1.0 \mathrm{TeV}$

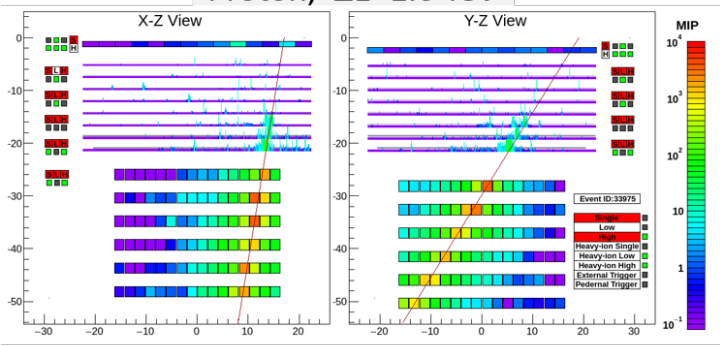

$\mathrm{Fe}(\mathrm{Z}=26), \Delta \mathrm{E}=1.2 \mathrm{TeV}$

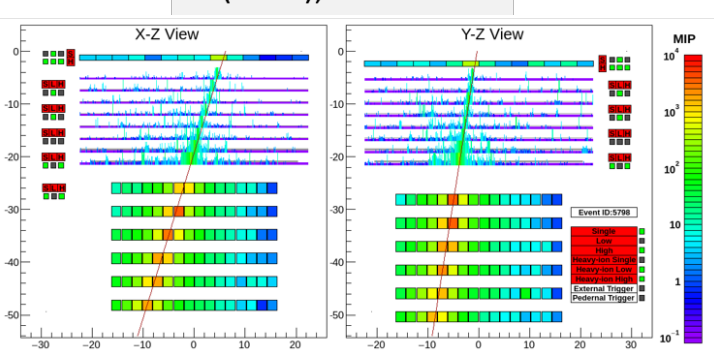

Gamma-ray, E=190 GeV

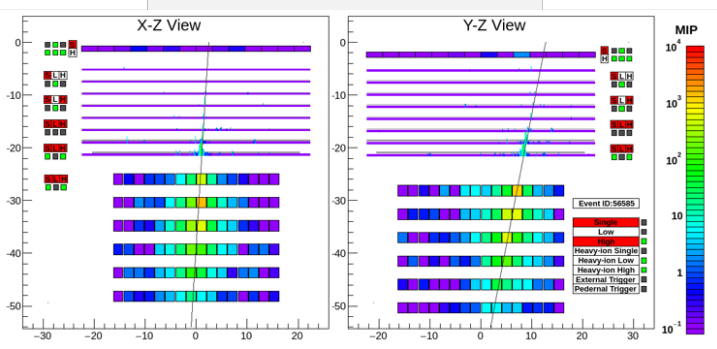

Figure 3. Event examples of high-energy showers collected by CALET onboard the ISS. From top to bottom, a $1 \mathrm{TeV}$ electron candidate, a proton candidate with equivalent shower energy, an iron candidate with shower energy of $1.2 \mathrm{TeV}$, and a $190 \mathrm{GeV}$ gamma-ray candidate, are shown respectively.

more than $10^{5}$, (2) a $2 \%$ energy resolution above $20 \mathrm{GeV}$ for electrons, (3) very wide dynamic range from $1 \mathrm{GeV}$ to $1 \mathrm{PeV}$, (4) charge resolution of $0.1-0.3$ electron charge unit from protons to over iron (up to $Z=40$ ). (5) an angular resolution of 0.1 to $0.5^{\circ}$, and (6) a large geometrical factor on the order of $0.1 \mathrm{~m}^{2} \mathrm{sr}$.

Figure 3 showcases high-energy shower events, summarizing the CALET capability of particle identification. From top to bottom, panels show a $1 \mathrm{TeV}$ electron candidate, a proton candidate with equivalent shower energy, an iron candidate with shower energy of $1.2 \mathrm{TeV}$, a gammaray candidate with $190 \mathrm{GeV}$ reconstructed energy, respec- 


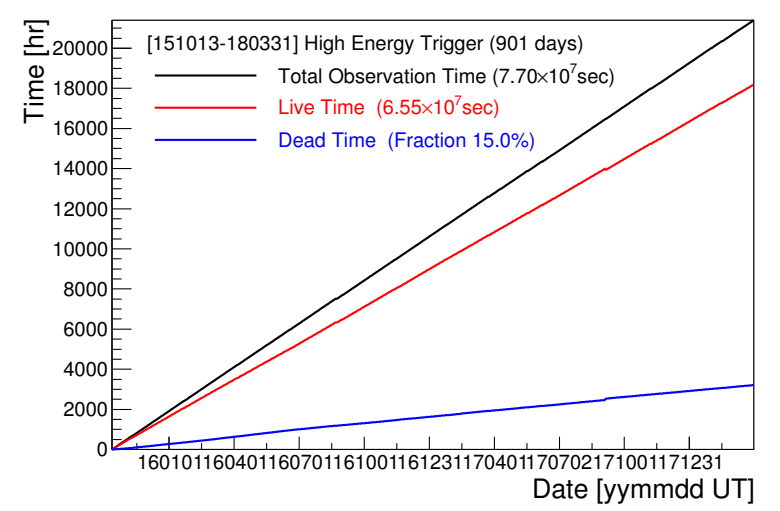

Figure 4. An accumulation of observation time (black line) for HE trigger. The red and blue lines indicate the live and dead times, respectively.

tively. The calorimeter with $30 X_{0}$ on-axis thickness absorbs the whole electron shower energy even in the $\mathrm{TeV}$ range. Charge measurement using CHD and IMC separates the each element from $Z=1$ to 26 and above. Gamma-rays are identified as charge zero because they do not produce any energy deposit before the pair creation. While both of electrons and protons have $Z=1$, they can be separated using shower shape differences. Because of the continuing showering activity in the lower part of the TASC caused by subsequent interactions of secondary pions, electrons and protons are easily separated by a simple cut even in the $\mathrm{TeV}$ region. In addition to this, various parameters characterizing the shower shape can be utilized to improve the separation power $[4,5]$.

\section{On-Orbit Operations}

The observation data obtained with CALET onboard ISS is transferred to JAXA via the NASA data-downlink system using the Tracking and Data Relay Satellite System. In order to operate the CALET onboard the ISS, JAXA Ground Support Equipment (JAXA-GSE) and the Waseda CALET Operations Center (WCOC) have been set up at Tsukuba Space Center and Waseda University, respectively. Scientific operations using CALET are planned at WCOC, taking into account variations of the geomagnetic rigidity cutoff with orbital position [6]. Scheduled command sequences are used to control the CALET observation modes on orbit. Calibration data acquisition by, for example, recording pedestal and penetrating particle events, a low-energy electron trigger mode operating at high geomagnetic latitude, a low-energy gamma-ray (LE$\gamma$ ) trigger mode operating at low geomagnetic latitude, and a nearly continuously operating ultra heavy trigger mode, are scheduled around the ISS orbit. Maximum exposure to high-energy electrons and other high-energy shower events is ensured by always having the high-energy (HE) trigger mode active.

As of March 31, 2018, the total observation time is 901 days with a live time fraction of the total time of $\sim 84 \%$. Nearly 590 million events are collected with HE $(E>10 \mathrm{GeV})$ trigger. Figure 4 shows the accumulated live time and number of events for the high-energy (HE) trigger. Since October, 2015, the observation time has increased smoothly without major interruption. Transmission of data from JAXA-GSE to WCOC and processing of the data for scientific analysis at WCOC have also proceeded smoothly.

\section{Calibration}

There is an intrinsic advantage in measuring the electromagnetic components of cosmic rays with CALET. Since the TASC absorbs the majority of the energy ( $95 \%)$ contained in an electromagnetic cascade, well into the $\mathrm{TeV}$ region, CALET is able to measure the primary energies of cosmic ray electrons and gamma rays with very small corrections. In principle, this should result in precise energy measurements with low systematic errors. However, in order to achieve a calibration accuracy that matches the intrinsic energy resolution over the wide dynamic range of six orders of magnitude, a careful calibration of each TASC readout channel is required.

The entire dynamic range is covered by four different gain ranges, based on two photon detectors - an APD and a PD - in conjunction with a shaper amplifier with lower and higher gains. Note that the first layer is read out by PMT for fast response required by the trigger. The energy calibration process [7] consists of three steps, i.e., (1) determination of the conversion factor between ADC units and the energy deposit, (2) linearity measurements over each gain range, and (3) correlation measurements between adjacent gain ranges.

The first step is the calibration of the energy deposit of each channel to obtain an ADC unit-to-energy conversion factor using MIPs, known as MIP calibration. As is the case with other detectors intended for direct cosmicray measurements, CALET can use penetrating particles to equalize the gains of different detector segments, based on the fact that the energy deposits of such particles in the relativistic energy range are approximately constant.

Prior to launch, the linearity over each gain range was confirmed by on-ground calibration using a UV pulse laser, during which the APD and PD outputs were determined as a function of the laser energy. In this process, the UV laser pulse was absorbed by the PWO, while the APD and PD detected the scintillation emissions resulting from de-excitation of the PWO. By scanning the UV laser pulses over the entire energy range, it was therefore possible to calibrate the input-to-output correspondence for all four gain ranges.

The adjacent gain ranges were subsequently crosscalibrated based on their gain ratios on orbit. Taking advantage of the nearly one order of magnitude overlap between adjacent gain ranges, it was possible to measure identical energy deposits within two gain ranges. As the linearity of each gain range had already been confirmed, the gain over the entire dynamic range could be determined based on the ADC-to-energy conversion factor, using the acquired gain ratios between adjacent gain ranges.

In addition, temporal gain variations occurring during long time observations are also corrected for in the cali- 

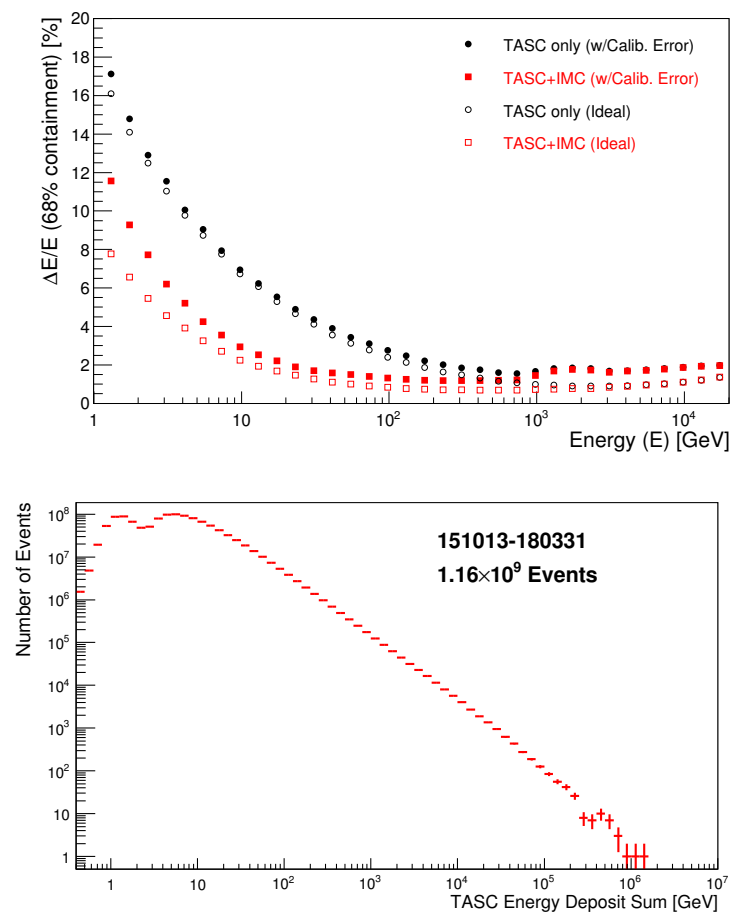

Figure 5. (Top) Energy resolution for electron measurements [7], (Bottom) TASC energy deposit spectrum using all triggered events up to March, 2018.

bration procedure [4]. As a consequence, our energy calibration provides a seamless transition between neighboring gain ranges, resulting in a very high resolution of $2 \%$ or better being achieved above $20 \mathrm{GeV}$ [7] as shown in the top panel of Fig. 5. It should be noted that even with such a detailed calibration, the determining factor for the energy resolution is the calibration uncertainty, as the intrinsic resolution of CALET is $\sim 1 \%$.

The bottom panel of Fig. 5 shows the TASC energy deposit spectrum using all triggered events through the end of March, 2018. The first bump is due to low-energy triggered events, while the second bump is caused by highenergy triggered events and the tail at the higher energy region reflects the power-law nature of the cosmic-ray spectrum. The spectrum spans more than six orders of magnitude in energy with highest energy past a $\mathrm{PeV}$, and the lowest energy below $1 \mathrm{GeV}$. This clearly demonstrates CALET's capability to observe cosmic rays over a very wide dynamic range.

\section{Results}

\subsection{All-Electron Spectrum}

A precise measurement of the all-electron (electron + positron) spectrum in the $\mathrm{TeV}$ region might reveal interesting spectral features to provide the first experimental evidence of the presence of a nearby cosmic-ray source $[8,9]$. In addition, the unexpected increase of the positron fraction over $10 \mathrm{GeV}$ established by PAMELA [10] and AMS02 [11] may require a primary source component for positrons in addition to the generally accepted secondary

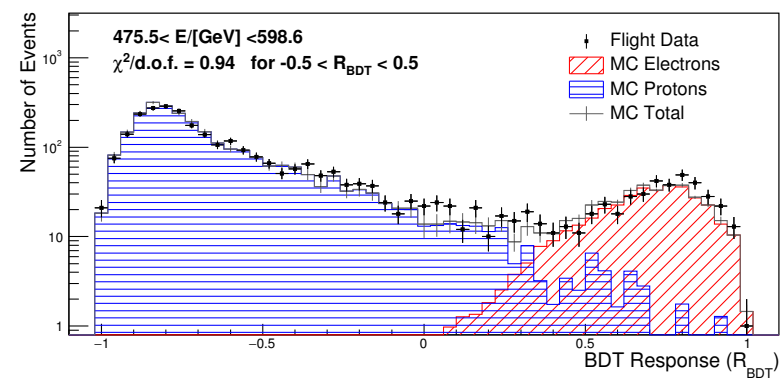

Figure 6. An example of BDT response distributions in the 476 $<E<599 \mathrm{GeV}$ bin including all acceptance conditions [5]

origin. Candidates for such primary sources range from astrophysical (pulsar) to exotic (dark matter). Since these primary sources emit electron-positron pairs, it is expected that the all-electron spectrum would exhibit a spectral feature, near the highest energy range of the primary component.

As of writing, the CALET collaboration published two papers regarding all-electron spectrum measurements [4, 5]. Electron spectrum analysis starts with track and energy reconstruction, followed by electron event selection and flux normalization [4]. In the first stage of event reduction, we selected well reconstructed shower events fully contained in the fiducial volume. Then, single charged particles were selected using energy deposits in the upper layers of the detector.

Finally, electron identification from large proton background was carefully done based on two methods using the shower shape difference in the detector: simple two parameter cuts and multivariate analysis based on boosted decision trees (BDTs), to understand systematic effects and the stability of the resultant flux. The details concerning these methods are explained in the Supplemental Material of Ref. [4]. An example of a BDT response distribution including all acceptance conditions, i.e., a geometrical factor of $0.1 \mathrm{~m}^{2} \mathrm{sr}$, is shown in Fig. 6. In the final electron sample, the resultant contamination ratios of protons are $\sim 5 \%$ up to $1 \mathrm{TeV}$, and $10 \%-20 \%$ in the $1-4.8 \mathrm{TeV}$ region, while keeping a constant high efficiency of $80 \%$ for electrons.

The geometrical acceptance and selection efficiencies were calculated using the detailed Monte Carlo simulation of the CALET flight model in which all the relevant detector responses were taken into account $[4,5,7]$. Various consistency checks between data and MC simulation were performed using flight data.

Figure 7 shows the updated all-electron spectrum [5] obtained with CALET using the same energy binning as in our first publication [4], except for adding one extra bin at the high energy end. The error bars along horizontal and vertical axes indicate bin width and statistical errors, respectively. The gray band is representative of the quadratic sum of statistic and systematic errors. The exhaustive study on the systematic uncertainties was per- 


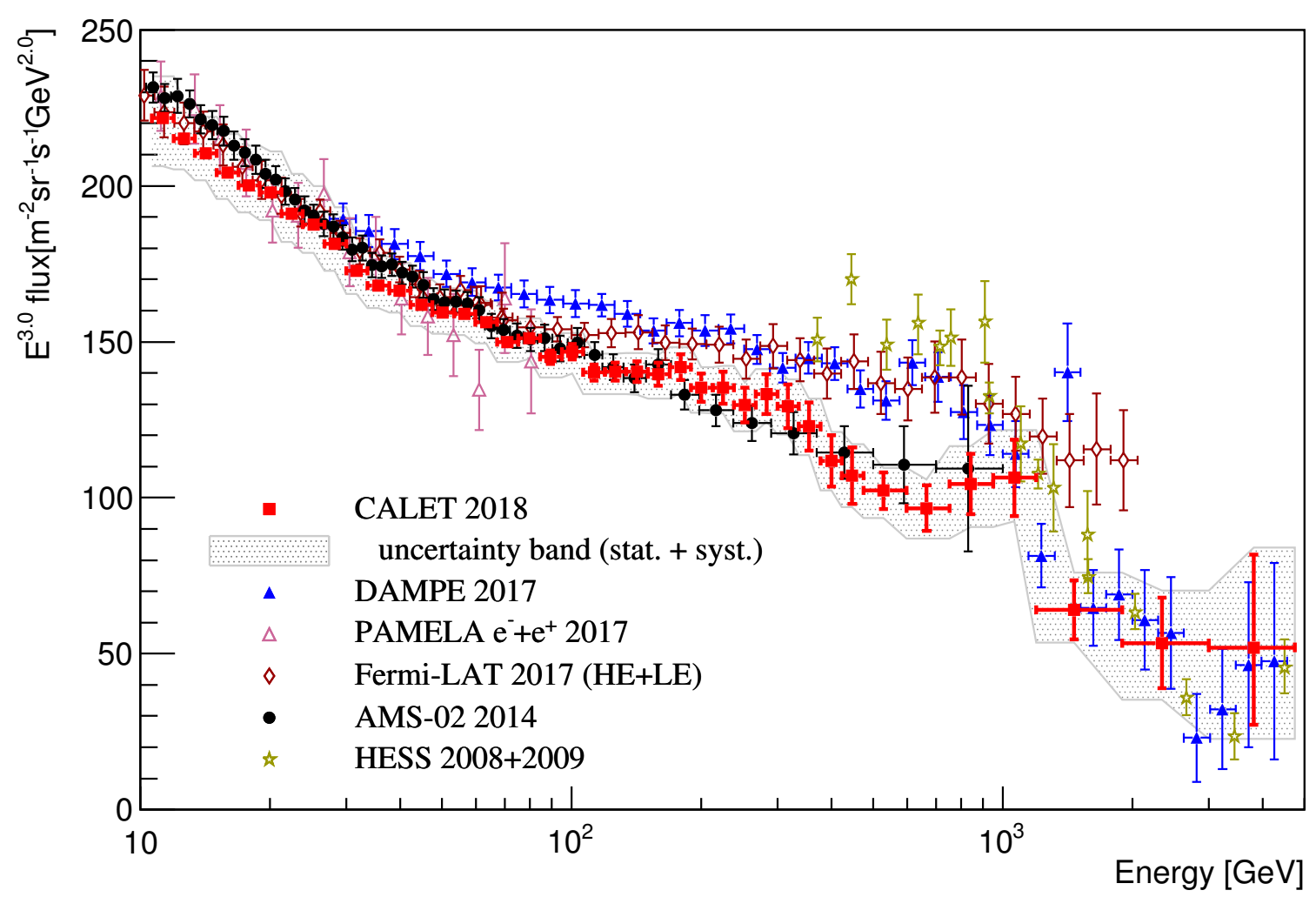

Figure 7. Cosmic-ray all-electron spectrum measured by CALET from $10.6 \mathrm{GeV}$ to $4.75 \mathrm{TeV}$ [5] using the same energy binning as in our first publication [4], where the gray band indicates the quadratic sum of statistical and systematic errors (not including the uncertainty on the energy scale). Also plotted are direct measurements in space [12-15] and from ground-based experiments [16, 17].

formed and is described in Refs. [4, 5] including their Supplemental Materials.

There are four important implications from the current status on the all-electron spectrum measurements. At first, CALET's spectrum is consistent with AMS-02 below $1 \mathrm{TeV}$. Because both detectors have enough capability to identify electrons up to $1 \mathrm{TeV}$, and because the detection principles are largely different (calorimeter versus magnet spectrometer), the agreement is an important indication. Secondly, there are two group of measurements: AMS$02+$ CALET vs Fermi-LAT + DAMPE, indicating the presence of unknown systematic errors. Thirdly, CALET observes flux suppression consistent with DAMPE within errors above $1 \mathrm{TeV}$. At last, however, no peak-like structure was found at $1.4 \mathrm{TeV}$ in CALET data, irrespective of energy binning. When using exactly the same binning as DAMPE, the flux at $1.4 \mathrm{TeV}$ bin is inconsistent between the two experiments with a 4 sigma significance [5]. As sharp structures in the spectrum may be blurred by binning effects, we studied them by shifting the energy binning and confirmed that such effects are negligible compared to our estimated systematic uncertainties [5].

\subsection{Gamma-Rays}

With its fully active $30 X_{0}$ thick calorimeter, CALET is capable of measuring gamma-rays up to the $\mathrm{TeV}$ region. In addition to HE trigger, CALET uses LE- $\gamma$ trigger to be sensitive to gamma rays with primary energies down to $1 \mathrm{GeV}$. To avoid large dead-time fraction, however, LE- $\gamma$ is activated only at low geomagnetic latitudes or following gamma-ray burst triggered onboard by CGBM.

The first 24 months of on-orbit scientific data provide valuable characterization of the performance of the calorimeter based on analyses of the gamma-ray data set [18]. It includes optimization of event selection criteria, calculation of effective area, determination of point spread function, confirmation of absolute pointing accuracy, observation of bright point sources and study of diffuse components.

Based on the developed analysis method, CALET gamma-ray sky seen by LE trigger is shown in the lefthand panel of Fig. 8, where galactic emission and bright gamma-ray sources are clearly identified. Point spread distributions and spectra of bright sources showed very good agreement with simulation and Fermi-LAT's results, respectively [18], confirming our sensitivity to observe GeV gamma-rays.

Gamma-ray transients are also an important observational target for CALET. CGBM detected nearly 60 GRBs ( $\sim 20 \%$ short GRB among them) per year in the energy range of $7 \mathrm{keV}-20 \mathrm{MeV}$, as expected [19]. To search for $\mathrm{GeV}$-energy counterpart emission from such sources also detected by other instruments, we check the calorimeter data at the reported trigger times for gamma-ray candidates. For events checked based on CGBM, Swift, and Fermi/GBM triggers, no significant counterparts have been detected at this stage of the analysis for timescales ranging from $1 \mathrm{~s}$ to $1 \mathrm{hr}$ [18]. Regarding the counterpart search for gravitational wave events, combined 


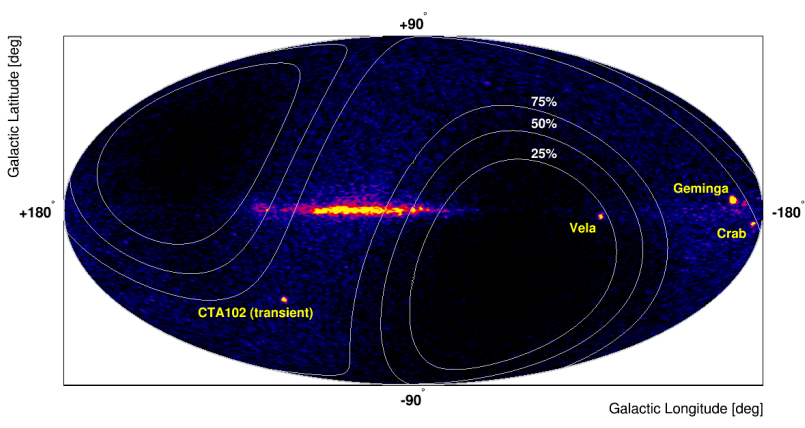

Figure 8. Gamma-ray sky map [18] shown in a Mollweide projection of galactic coordinates. White contours show the relative level of exposure compared to the maximum on the sky. The Crab, Geminga, and Vela pulsar are clearly visible, as is a flare of the AGN CTA 102.

analysis of CGBM and calorimeter was performed for GW151226 setting our upper limits on X-ray and gammaray counterparts [20]. Furthermore, complete search results of CALET calorimeter on the LIGO/Virgo's Observation Run 2 was recently published [21].

\subsection{Hadrons}

Direct measurements of the high-energy spectra of each cosmic-ray nuclei species up to the PeV energy scale provide insight into the general conditions of cosmic-ray acceleration and propagation, complementing information from all-electron observations. A charge-dependent cutoff in the nuclei spectra might explain the "knee" in the all-particle spectrum, a hypothesis only explorable by a space experiment with sufficient exposure. The calculated acceleration limit of supernova remnants with nominal parameters is typically found to be far smaller than the indirectly observed energy of the "knee" [22], calling for precise direct observation of the proton and helium spectra up to PeV energy. The spectral hardening observed for various nuclei can be well studied taking advantage of CALET's wide dynamic range from $\mathrm{GeV}$ to $\mathrm{PeV}$ energies, avoiding systematics from combination of spectra measured by different experiments. Another detailed study focuses on the spectral behavior of heavier elements, including secondary-to-primary ratios up to the $1 \mathrm{TeV} / \mathrm{n}$ energy region, providing information on propagation parameters such as the diffusion coefficient.

Figure 9 demonstrates the charge identification capability of CALET only with CHD [23], where clear separation of protons, helium and heavier nuclei up to iron and nickel is presented. IMC adds similar and independent charge separation power for light elements. Based on this accurate charge determination and wide energy range (See Fig. 5), preliminary results on protons [24] and heavier nuclei $[23,25]$ are presented until now. The first results on nuclei will be published soon, including the detailed assessment of systematic uncertainties.

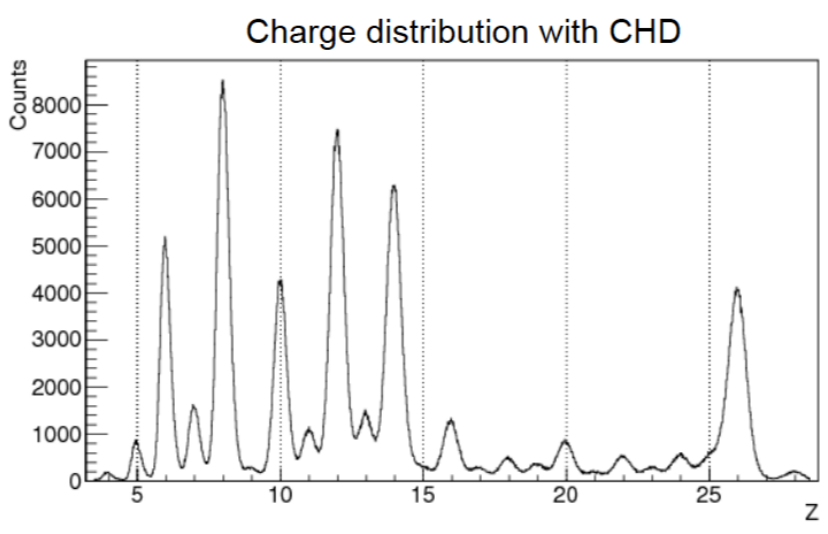

Figure 9. CHD charge separation. Each element is clearly identified up to Nickel [23] (The CHD dynamic range covers up to $Z=40)$.

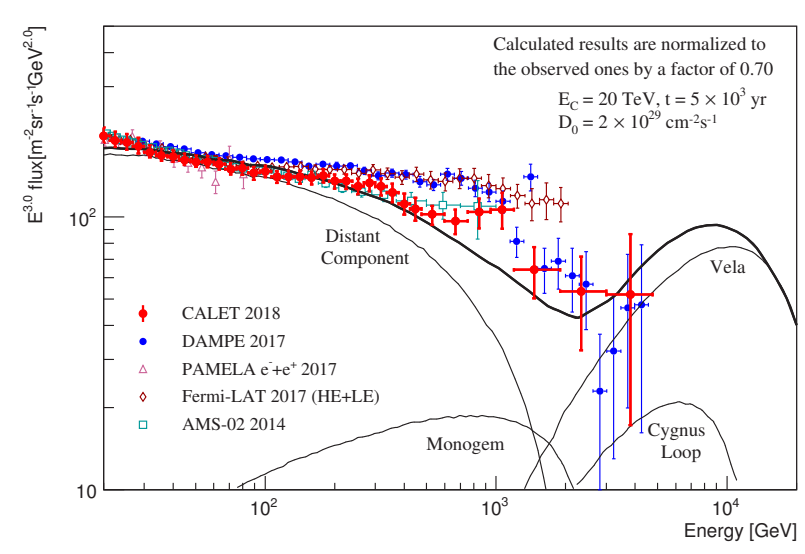

Figure 10. Current situation of all-electron spectrum directly measured in space, together with the model calculation [9], where important model parameters are shown in the plot.

\section{Summary and Prospects}

CALET was successfully launched on Aug. 19, 2015, and the detector has been continuously very stable since Oct. 13, 2015. Careful calibrations have been performed by using "MIP" signals of the non-interacting protons and helium events, and the linearity in the energy measurements up to $10^{6}$ MIPs is confirmed based on observed events $[6,7]$.

All electron spectrum [4] results have been extended in statistics and in the energy range, which is now from 11 $\mathrm{GeV}$ to $4.8 \mathrm{TeV}$ [5]. Figure 10 shows the current situation of the all-electron spectrum direct measurements in space. Precision will greatly increase with five or more years of CALET observation, due to tripled statistics and reduced systematic errors from continued recording of flight data leading to a better understanding of the detector.

This will allow a refined study of the possible fine structures around a few hundred $\mathrm{GeV}$ and $\sim 1 \mathrm{TeV}$, which are currently not significant, but if confirmed it would shed light on the origin of the positron excess. An extension of the high-energy reach using improved statistics and elec- 
tron separation methods might for the first time reveal a charged cosmic-ray signature of a local accelerator.

Regarding hadron analysis, its wide dynamic range of energy measurement from $1 \mathrm{GeV}$ to $1 \mathrm{PeV}$ and accurate charge determination capability are two major advantages of CALET. Based on these abilities, preliminary results on protons [24], primary and secondary nuclei spectra up to $Z=26$, and their ratios (for example, boron to carbon) [23, 25] have been presented so far. They will be published in turn and will address important questions in cosmic-ray physics like charge dependence of the acceleration limit in supernova remnants, universality and/or detailed behavior of the widely observed hardening in nuclei spectra, and energy dependence of the diffusion coefficients. Analysis on the relative abundance of the ultra heavy nuclei above $Z=28$ through $Z=40$ is also in progress [26].

CALET's gamma-ray analysis has also made significant progress. Based on the two-year science data onorbit, the performance of the gamma-ray measurements is characterized [18]. These results establish the capability of CALET to observe gamma rays in the energy range from $\sim 1 \mathrm{GeV}$ to over $100 \mathrm{GeV}$. Current results on the electromagnetic counterpart search for gravitational wave events by CALET $[20,21]$ indicate many more results during the coming LIGO/Virgo's Observation Run 3. Monitoring the $\mathrm{GeV}$ sky with CALET may complement the coverage by other missions and may help to study unexplored high-energy emission from future transient events. As a working instrument on orbit, watching for various transient phenomena, including but not limited to gammarays, is an important task to be actively pursued. By detecting many REP events [27], where REP stands for relativistic electron precipitation, space weather became an observational target of CALET since the start of on-orbit operations.

The so far excellent performance of CALET and the outstanding quality of the obtained data suggest that a 5year (or more) observation period is likely to provide a wealth of interesting new results.

\section{Acknowledgment}

We gratefully acknowledge JAXA's contributions to the development of CALET and to the operations onboard the International Space Station. We also wish to express our sincere gratitude to ASI and NASA for their support of the CALET project. This work was supported in part by JSPS Grant-in-Aid for Scientific Research (S) (No. 26220708), JSPS Grant-in-Aid for Scientific Research (B) Number 17H02901, JSPS Grant-in-Aid for Scientific Research (C) Number 16K05382, and by the MEXT-Supported Program for the Strategic Research Foundation at Private Universities (2011-2015) (No. S1101021) at Waseda University. The CALET effort in the United States is supported by NASA through Grants No. NNX16AB99G, No. NNX16AC02G, and No. NNH14ZDA001N-APRA-0075.

\section{References}

[1] S. Torii et al. (CALET Collaboration), in Proceeding of Science (ICRC2015) 581 (2015)

[2] S. Torii et al. (CALET Collaboration), in Proceeding of Science (ICRC2017) 1092 (2017)

[3] K. Yamaoka et al., in Proc. 7th Huntsville GammaRay Burst Symposium, GRB 2013 (2013), p. 41

[4] O. Adriani et al. (CALET Collaboration), Phys. Rev. Lett. 119, 181101 (2017)

[5] O. Adriani et al. (CALET Collaboration), Phys. Rev. Lett. 120, 261102 (2018)

[6] Y. Asaoka, Y. Ozawa, S. Torii et al. (CALET Collaboration), Astropart. Phys. 100, 29 (2018)

[7] Y. Asaoka, Y. Akaike, Y. Komiya, R. Miyata, S. Torii et al. (CALET Collaboration), Astropart. Phys. 91, 1 (2017)

[8] J. Nishimura et al., ApJ 238, 394 (1980)

[9] T. Kobayashi, Y. Komori, K. Yoshida, J. Nishimura, ApJ 601, 340 (2004)

[10] O. Adriani et al., Nature 458, 607 (2009)

[11] L. Accardo et al. (AMS Collaboration), Phys. Rev. Lett. 113, 121101 (2014)

[12] G. Ambrosi et al. (DAMPE Collaboration), Nature 552, 63 (2017)

[13] O. Adriani et al., La Rivista del Nuovo Cimento 40, 473 (2017)

[14] S. Abdollahi et al. (The Fermi-LAT Collaboration), Phys. Rev. D 95, 082007 (2017)

[15] M. Aguilar et al. (AMS Collaboration), Phys. Rev. Lett. 113, 221102 (2014)

[16] F. Aharonian et al. (H.E.S.S. Collaboration), Phys. Rev. Lett. 101, 261104 (2008)

[17] F. Aharonian et al. (H.E.S.S. Collaboration), Astron Astrophys. 508, 561 (2009)

[18] N. Cannady, Y. Asaoka et al. (CALET Collaboration), ApJS 238, 5 (2018)

[19] K. Yamaoka et al. (CALET Collaboration), in Proceeding of Science (ICRC2017) 613 (2017)

[20] O. Adriani et al. (CALET Collaboration), ApJL 829, L20 (2016)

[21] O. Adriani et al. (CALET Collaboration), ApJ 863, 160 (2018)

[22] A.R. Bell, K.M. Schure, B. Reville, G. Giacinti, MNRAS 431, 415 (2013)

[23] Y. Akaike et al. (CALET Collaboration), in Proceedings of Science (ICRC2017) 181 (2017)

[24] P.S. Marrocchesi et al. (CALET Collaboration), in Proceeding of Science (ICRC2017) 156 (2017)

[25] Y. Akaike et al. (CALET Collaboration), in this conference (2018)

[26] R. Brian, Y. Akaike et al. (CALET Collaboration), in Proceeding of Science (ICRC2017) 180 (2017)

[27] R. Kataoka et al., Geophys. Res. Lett. 43, doi:10.1002/2016GL068930 (2016) 